\title{
Hemarthrosis Resulting from Venous Thromboembolism Prophylaxis in the Postoperative Ambulatory Orthopedic Sports Medicine Patient: A Retrospective Study of Rivaroxaban Versus Enoxaparin
}

\author{
Brian P Davis $^{1 *}$, Darshan Patel ${ }^{1}$, Michelle M Coleman ${ }^{1}$ and Anup Shah ${ }^{2}$ \\ ${ }^{1}$ Department of Orthopedic Surgery, Baylor College of Medicine, Houston, USA \\ ${ }^{2}$ Kelsey-Seybold Clinic, West Holcombe Blvd, Houston, USA \\ *Corresponding Author: Brian P Davis, Department of Orthopedic Surgery, Baylor \\ College of Medicine, Houston, USA.
}

Received: September 20, 2021

Published: October 04, 2021

(C) All rights are reserved by Brian P Davis., et al.

\begin{abstract}
Purpose: The purpose of this study was determining the risk of hemarthrosis associated with prophylactic administration of rivaroxaban and enoxaparin in an ambulatory postoperative sports medicine patient population after knee arthroscopic procedures.

Methods: A retrospective observational chart review was performed for 214 patients [aged 16 to 53] who underwent arthroscopic knee surgery performed at a single surgical center between November 2011 and December 2016 and received postoperative VTE chemoprophylaxis. All patients were prescribed postoperative thromboprophylaxis with either rivaroxaban (dosed 10 mg orally every 24 hours for ten days) or enoxaparin (dosed 40mg subcutaneously every 24 hours for ten days). These patients were followed for a minimum three-month period post-operatively. All patients were evaluated at two and six weeks postoperatively and joint aspiration was performed if patients presented with painful and clinically significant knee swelling/effusion (grade 2+ to 3+) and/or limited range of motion. Deep vein thrombosis was ruled out by Doppler ultrasound if clinically suspected. Patients were excluded if age was less than 16 years or were not prescribed postoperative thromboprophylactic medication.
\end{abstract}

Results: Our data revealed that while there was no incidence of DVT in either group, 12.5\% of patients in the rivaroxaban cohort were found to have clinically significant hemarthrosis versus $1.6 \%$ of patients in the enoxaparin group (Odds Ratio 8.9 ; CI 1.912 41.034, p-value $=0.0053$ ).

Conclusion: If a surgeon chooses to prescribe chemoprophylaxis for ambulatory postoperative sports medicine patients, this study supports consideration of avoiding rivaroxaban, given the higher risk of a symptomatic hemarthrosis in the early postoperative period.

Keywords: Arthroscopic Knee; Hemarthrosis; Sports Medicine; Thromboprophylaxis; Venous Thromboembolism

\section{Introduction}

The incidence of deep venous thrombosis (DVT) and/or pulmonary embolus (PE) after arthroscopic knee surgery is estimated to be approximately $10 \%$, with rates varying from $0.02 \%$ to $41.2 \%$ in individual studies [1-13]. This wide variation is in part due to heterogeneous patient populations, diagnostic methodologies, and 
varied surgical procedures. With greater than 100,000 anterior cruciate ligament reconstructions performed in the Unites States annually [6], the volume of symptomatic DVT in this population must be recognized, treated and, ideally, prevented to improve patient safety.

The decision to prophylactically treat the ambulatory sports medicine patient with chemoprophylaxis currently resides with surgeon discretion. Risk factors such as age greater than 40 years, obesity with a body mass index greater than $30 \mathrm{~kg} / \mathrm{m}^{2}$, current oral contraceptive use, thrombophilic disorders or history of malignancy, personal/family history of DVT, nicotine use, prolonged tourniquet time over 90 - 120 minutes, and presence of two or more risk factors affect the decision-making process [2,3,13-15]. Traditionally, various DVT/VTE prophylactic agents have been prescribed in elective total hip or knee arthroplasty including low molecular weight heparin (LMWH), enoxaparin (Lovenox ${ }^{\circledR}$, SanofiAventis U.S. LLC, Bridgewater, New Jersey) and more recently, rivaroxaban (Xarelto ${ }^{\circledR}$, Janssen Ortho LLC, Gurabo, Puerto Rico) [8]. The American Academy of Orthopaedic Surgeons (AAOS) has provided guidelines for routine prophylaxis after elective total joint arthroplasty [16], however no guidelines have been developed for patients undergoing elective arthroscopic knee procedures. The 2012 American College of Chest Physicians (ACCP) recommendation for patients undergoing arthroscopic knee surgery (inclusive of ACL reconstruction, meniscectomies, and other diagnostic and therapeutic arthroscopic procedures) was to not prescribe routine thromboprophylaxis for patients without a history of prior VTE [4]. However, the authors stated that the studies included in the systematic review were of low-quality and did provide evidence that low molecular weight heparin (LMWH) can reduce DVT and VTE in this patient population. They did recommend thromboprophylaxis with LMWH for patients with VTE risk factors. They also noted that there was a potential risk for major bleeding events (defined as hemorrhage associated with transfusion, hemorrhage requiring surgical intervention, or resulting in permanent disability) after LMWH use with an estimated incidence of 3 per 1,000 treated patients [4]. Several studies have shown the efficacy of both low molecular weight heparin (LMWH) and rivaroxaban in knee arthroscopy and ACL reconstruction [5]. However, few studies have elucidated whether one is preferable for the ambulatory surgical patient.

\section{Purpose of the Study}

The purpose of the current study was to determine the risk of hemarthrosis associated with prophylactic administration of rivaroxaban and enoxaparin in an ambulatory postoperative sports medicine patient population after knee arthroscopic procedures. The null hypothesis was there would be no difference in the incidence of DVT and adverse reactions with both agents.

\section{Methods}

\section{Study design}

This was a retrospective observational chart review performed at a single surgical center with all surgical procedures performed by a single orthopedic sports medicine fellowship-trained surgeon and senior author. The Institutional Review Board approved the study protocol prior to data collection. The study was conducted according to principles as stated in the Declaration of Helsinki. This research did not receive any specific grant from funding agencies in the public, commercial, or not-for-profit sectors.

Inclusion criteria included patients of 16 to 55 years of age who were scheduled for common orthopedic sports medicine procedures, namely ACL reconstruction, meniscus repair, and/or chondral surgery. Exclusion criteria included age less than 16 years old (patients prior to physeal closure and skeletal maturity), follow up duration less than three months, and patients who underwent knee arthroscopic procedures and did not receive postoperative thromboprophylaxis with enoxaparin or rivaroxaban for 10 days. Given the lack of formal recommendations on chemoprophylactic agent, quantity, or duration of treatment, the decision for chemoprophylaxis was based on risk factors associated with the surgical procedure performed and concern for lack of postoperative mobilization at the surgeon's discretion. The primary surgeon's indications for postoperative thromboprophylaxis included personal history of prior VTE, family history of VTE, current smoker, current oral contraceptive use, obesity (body mass index greater than 30 $\mathrm{kg} / \mathrm{m}^{2}$ ), prolonged non-weight bearing or immobilization (for four or more weeks), and lack of patient mobilization due to pain or patient non-compliance with rehabilitation protocols.

Patients were sequentially divided into two groups over a fiveyear period. The time period for data collection was from November 2011 to November 2016. The first group of patients were pre- 
Hemarthrosis Resulting from Venous Thromboembolism Prophylaxis in the Postoperative Ambulatory Orthopedic Sports Medicine Patient: A Retrospective Study of Rivaroxaban Versus Enoxaparin

scribed oral rivaroxaban (Xarelto ${ }^{\circledR}$ ) $10 \mathrm{mg}$ dosed every 24 hours starting the morning after surgery for a duration of ten days as DVT chemoprophylaxis during the time period from November $28^{\text {th }}$, 2011 to December $30^{\text {th }}, 2014$. The second group was treated with subcutaneously injected enoxaparin (Lovenox ${ }^{\circledR}$ ) 40mg dosed every 24 hours starting the morning after surgery for a duration of ten days during the time period from January $14^{\text {th }}, 2015$ to December $29^{\text {th }}, 2016$. If a patient had other risk factors aside from a personal history, a hematologist's recommendations were followed for postoperative thromboprophylaxis.

All patients were treated at a single outpatient surgery center and were discharged home the same day. All patients received compression stockings and a hinged knee brace to wear postoperatively. All patients were restricted to non-weight bearing on the affected extremity for 4 - 6 weeks. Patients who underwent ACL reconstruction or meniscal repair or partial meniscectomy started a passive and active range of motion program within two to three days of surgery.

All patients were routinely evaluated in clinic at two and six weeks postoperatively by the operative surgeon or a physician assistant with careful attention for adverse effects, with special attention to signs or symptoms of DVT and/or hemarthrosis. Patients were advised to schedule an immediate clinic visit if knee pain or effusion worsened or they experienced signs or symptoms of DVT. Minimum length of follow-up was three months postoperatively. Providers were not blinded to the method of prophylaxis. Painful knees with $2+$ and $3+$ knee effusions were aspirated under sterile conditions in the clinic setting based on the patient's pain assessed by visual analog score (range from 0 - 5) and clinical evaluation of knee effusion graded using the Clinical Effusion Rating Scale as defined by Sturgill., et al. [17] (See table 1). This grading scale is based upon clinical examination using a stroke test in an attempt to mobilize fluid from the medial joint line upwards toward the suprapatellar pouch followed by a second downward stroke from superolateral to the lateral joint line. Fluid waves and shifts are observed and palpated. This test has been shown to have improved interrater reliability as compared to the fluctuation test or patellar tap test [17]. All postoperative knees that underwent aspiration were in patients who subjectively reported pain (visual analog score of 1 or higher) and had 2+ to 3+ knee effusion on clinical exam.

\begin{tabular}{|l|c|}
\hline Grade & Test Result \\
\hline 0 & No wave produced on downstroke \\
\hline Trace & Small wave on medial side of knee with downstroke \\
\hline $1+$ & Larger bulge on medial side of knee with downstroke \\
\hline $2+$ & $\begin{array}{c}\text { Effusion spontaneously returns to medial side after } \\
\text { upstroke }\end{array}$ \\
\hline $3+$ & $\begin{array}{c}\text { Not possible to move fluid out of medial aspect of } \\
\text { knee }\end{array}$ \\
\hline
\end{tabular}

Table 1: Clinical effusion rating scale, adapted and reproduced with permission from Sturgill LP, Snyder-Mackler L, Manal TJ, Axe MJ. Interrater reliability of a clinical scale to assess knee joint effusion. J Orthop Sports Phys Ther 2009;39(12):845-849.

\section{Outcome evaluation}

The primary outcome of this study was the incidence of clinically significant hemarthrosis within a three-month period postoperatively. Clinically significant hemarthrosis was defined as a swollen, painful knee with clinical effusion rated as above that limited patient mobilization with arthrocentesis yielding a sanguineous aspirate. For patients who underwent arthrocentesis for symptomatic hemarthrosis, the postoperative date of aspiration was recorded as well as patient risk factors for DVT, visual analog pain score prior to aspiration, and amount of fluid aspirated. All aspirations were sent for routine synovial analysis, and no patient included in the study was diagnosed with a postoperative infection.

The secondary outcomes of the study were the rates of symptomatic DVT and PE. DVT was defined in patients with clinical findings of a painful and swollen lower extremity with a subsequent positive Doppler ultrasound for DVT. PE was defined in patients with symptoms consistent with PE (shortness of breath and/or tachycardia) and a diagnostic CT/PE or VQ scan.

\section{Follow-up}

Patients were followed for a period of one year postoperatively. Patients were instructed to contact the study center immediately if the developed new or worsening symptoms. All patients were followed up in clinic at two, six, sixteen, thirty weeks, and 52 weeks, during which time their health status, history of any hospital admissions, and any other concerns were updated using a standard questionnaire. 
Hemarthrosis Resulting from Venous Thromboembolism Prophylaxis in the Postoperative Ambulatory Orthopedic Sports Medicine Patient: A Retrospective Study of Rivaroxaban Versus Enoxaparin

\section{Statistical analysis}

Fisher's exact test and logistic regression analysis were used to assess our null hypothesis that there was no difference in aspiration/hemarthrosis risk in either treatment arm. A power analysis was performed and it was determined that in order to detect a DVT rate difference of eight percent, the study would require 91 patients in each cohort (total $n=182$ ). Similarly, in order to detect a hemarthrosis rate difference of twelve percent, the study would require 61 patients in each group (total $n=122$ ). Fisher's exact test and the Mann-Whitney test were utilized to compare demographic data between the two groups.

\section{Results}

Between November 2011 and December 2016, a total of 214 patients were eligible to be included in the study based on inclusion criteria. The study cohort consisted of 88 patients in the rivaroxaban group and 126 patients in the enoxaparin group. There were no statistically significant demographic differences between groups (See table 2).

\begin{tabular}{|l|c|c|c|}
\hline Characteristic & $\begin{array}{c}\text { Rivaroxaban } \\
\text { cohort (n, \%) }\end{array}$ & $\begin{array}{c}\text { Enoxaparin } \\
\text { cohort (n, \%) }\end{array}$ & P-value \\
\hline Age (years) & $32(36.4 \%)$ & $40(31.7 \%)$ & 0.52 \\
\hline $16-20$ & $24(27.2 \%)$ & $42(33.3 \%)$ & \\
\hline $21-30$ & $24(27.2 \%)$ & $25(19.8 \%)$ & \\
\hline $31-40$ & $8(9.1 \%)$ & $16(12.7 \%)$ & \\
\hline $41-50$ & - & $3(2.3 \%)$ & \\
\hline $51-55$ & $61(69.3 \%)$ & $81(64.3 \%)$ & 0.47 \\
\hline Gender & $27(30.7 \%)$ & $45(35.7 \%)$ & \\
\hline Male & $27.1 \pm 5.3$ & $27.0 \pm 5.1$ & 0.98 \\
\hline Female & & & \\
\hline BMI (kg/m $\left.{ }^{2}\right)$ & $88(100 \%)$ & $126(100 \%)$ & 0.99 \\
\hline ASA classification & $6(6.8 \%)$ & $13(10.3 \%)$ & 0.47 \\
\hline ASA 1 & $17(19.3 \%)$ & $19(15.1 \%)$ & 0.46 \\
\hline Oral contraceptive & & & \\
\hline use & & & \\
\hline Tobacco use & & & \\
\hline
\end{tabular}

Table 2: Comparison of categorical data between cohorts showed no statistical difference between groups. BMI = Body Mass Index, ASA = American Society of Anesthesiologists.
The percentage of surgical procedures performed in each cohort is listed in table 3 . The majority of the surgical procedures were ACL reconstructions with a concomitant meniscal repair or partial meniscectomy (128 procedures, 59.8\%), with the second most common procedure being meniscal repair or partial meniscectomy (26 procedures, 12.1\%). No surgeries had a tourniquet time greater than two hours.

\begin{tabular}{|l|c|c|c|c|}
\hline $\begin{array}{l}\text { Primary } \\
\text { procedure }\end{array}$ & $\begin{array}{c}\text { Rivaroxaban } \\
\text { cohort (n) }\end{array}$ & $\begin{array}{c}\text { Enoxaparin } \\
\text { cohort (n) }\end{array}$ & $\begin{array}{c}\text { Total } \\
\text { (n) }\end{array}$ & $\begin{array}{c}\text { Percent } \\
\text { (\%) }\end{array}$ \\
\hline $\begin{array}{l}\text { Primary ACL } \\
\text { reconstruction }\end{array}$ & 69 & 86 & 155 & $72.4 \%$ \\
\hline $\begin{array}{l}\text { Revision ACL } \\
\text { reconstruction }\end{array}$ & 2 & 10 & 12 & $5.6 \%$ \\
\hline $\begin{array}{l}\text { Other arthroscopic } \\
\text { procedure* }\end{array}$ & 17 & 30 & 47 & $22.0 \%$ \\
\hline Totals & 88 & 126 & 214 & $100.0 \%$ \\
\hline
\end{tabular}

Table 3: Primary surgical procedures performed for each cohort prescribed either enoxaparin or rivaroxaban for postoperative thromboprophylaxis. Secondary procedures not included for table simplicity. There was no statistical significance between these groups. *Other arthroscopic procedures include chondroplasty, meniscal repair or partial meniscectomy, other ligamentous repair or reconstruction (medial collateral or lateral collateral ligamentous structures), or a combination of these. Chondroplasty indicates procedures including microfracture or osteochondral allograft transplantation. Other ligamentous repair indicates repair or reconstruction of the medial collateral or lateral collateral ligamentous structures. ACL = Anterior Cruciate Ligament.

Of the total 214 patients included in the study, 13 patients underwent aspiration for diagnosis of hemarthrosis. All aspirations were performed between postoperative days 4 - 14. All patients who underwent aspiration had a Doppler ultrasound performed without evidence of DVT. There were ten males and three female patients who underwent aspiration (Table 4). No patient had a personal or family history of DVT. Two patients were smokers and one female patient was taking oral contraception.

Aspiration with resultant diagnosis of hemarthrosis was performed in eleven patients $(12.5 \%)$ who had been prophylacti- 
Hemarthrosis Resulting from Venous Thromboembolism Prophylaxis in the Postoperative Ambulatory Orthopedic Sports Medicine Patient: A Retrospective Study of Rivaroxaban Versus Enoxaparin

08

\begin{tabular}{|c|c|c|c|c|c|c|c|}
\hline $\begin{array}{c}\text { Anticoagulant } \\
\text { Therapy }\end{array}$ & Patient & $\begin{array}{c}\text { Age } \\
\text { (years) }\end{array}$ & Sex & Procedure Performed & $\begin{array}{l}\text { Aspiration } \\
\text { POD }\end{array}$ & $\begin{array}{l}\text { Effusion } \\
\text { rating }\end{array}$ & $\begin{array}{l}\text { Pain Score } \\
\text { (VAS) }\end{array}$ \\
\hline \multirow{11}{*}{ Rivaroxaban } & 1 & 36 & M & $\begin{array}{l}\text { Left knee ACL reconstruction (Achilles } \\
\text { allograft) + lateral meniscus repair (inside } \\
\text { out), medial femoral condyle chondroplasty }\end{array}$ & 4 & $3+$ & 5 \\
\hline & $2^{*}$ & 27 & M & $\begin{array}{l}\text { Left knee ACL reconstruction (Achilles al- } \\
\text { lograft) + medial meniscus repair (inside } \\
\text { out) }\end{array}$ & 6 & $2+$ & 4 \\
\hline & 3 & 29 & M & $\begin{array}{l}\text { Right knee ACL reconstruction (hamstring } \\
\text { autograft) + medial femoral condyle micro- } \\
\text { fracture }\end{array}$ & 4 & $2+$ & 4 \\
\hline & $4^{\wedge}$ & 16 & $\mathrm{~F}$ & $\begin{array}{c}\text { Left knee ACL reconstruction (bone-patellar } \\
\text { tendon-bone autograft) + medial meniscus } \\
\text { repair (bucket handle) }\end{array}$ & 8 & $2+$ & 3 \\
\hline & 5 & 36 & M & $\begin{array}{c}\text { Right knee ACL reconstruction (Achilles } \\
\text { allograft) + medial meniscus repair + partial } \\
\text { lateral meniscectomy }\end{array}$ & 6 & $3+$ & 4 \\
\hline & $6^{*}$ & 25 & M & Left knee medial meniscal repair & 8 & $2+$ & 5 \\
\hline & 7 & 17 & M & Left knee medial meniscal repair & 6 & $3+$ & 5 \\
\hline & 8 & 18 & M & $\begin{array}{c}\text { Right knee ACL reconstruction (bone-patel- } \\
\text { lar tendon-bone autograft) + bilateral partial } \\
\text { medial and lateral meniscectomies }\end{array}$ & 14 & $2+$ & 1 \\
\hline & 9 & 28 & M & Left knee lateral meniscal repair & 6 & $2+$ & 4 \\
\hline & 10 & 22 & $\mathrm{~F}$ & Left knee lateral meniscus saucerization & 9 & $2+$ & 2 \\
\hline & 11 & 20 & M & $\begin{array}{l}\text { Left knee medial meniscal repair + patellar } \\
\text { chondroplasty }\end{array}$ & 7 & $3+$ & 5 \\
\hline \multirow[t]{2}{*}{ Enoxaparin } & 1 & 25 & M & $\begin{array}{c}\text { Right knee ACL reconstruction (bone- } \\
\text { patellar tendon- bone autograft) + lateral } \\
\text { meniscus repair }\end{array}$ & 8 & $2+$ & 4 \\
\hline & 2 & 16 & $\mathrm{~F}$ & Left knee lateral meniscus repair & 7 & $2+$ & 4 \\
\hline
\end{tabular}

Table 4: Postoperative aspirated patient characteristics. Patient characteristics for patients with hemarthrosis after aspiration. ${ }^{\wedge}$ Indicates on oral contraceptives. ${ }^{*}$ Indicates current smoker. POD = Postoperative Day, VAS = Visual Analog Score, ACL = Anterior Cruciate Ligament, $\mathrm{M}=$ Male, $\mathrm{F}=$ Female.

cally anticoagulated with rivaroxaban compared with two patients $(1.6 \%)$ in the enoxaparin group (Figure 1). The odds of requiring aspiration were 8.9 times greater among patients prophylactically treated with rivaroxaban versus those treated prophylactically with enoxaparin ( $p=0.0053$; 95\% CI: 1.912 - 41.034, Table 5).

There were no DVTs or PEs diagnosed in either study group.

\section{Discussion}

Deciding when and how to prophylactically anticoagulate postoperative ambulatory orthopedic sports medicine patients is a controversial topic. A recent 2016 survey of 142 orthopedic surgeons discovered that $50.7 \%$ routinely used chemoprophylaxis after ACL reconstruction [18]. The most commonly prescribed prophylaxis was aspirin (95\%) with great variation in dosing and duration of 
Hemarthrosis Resulting from Venous Thromboembolism Prophylaxis in the Postoperative Ambulatory Orthopedic Sports Medicine Patient: A Retrospective Study of Rivaroxaban Versus Enoxaparin

\begin{tabular}{|l|c|c|c|c|c|}
\hline Anticoagulant & Total patients (\%) & $\begin{array}{c}\text { Patients with } \\
\text { hemarthrosis (\%) }\end{array}$ & $\begin{array}{c}\text { Patients without } \\
\text { hemarthrosis (\%) }\end{array}$ & P value & Odds Ratio (95\% CI) \\
\hline Rivaroxaban & $88(41.1)$ & $11(12.5)$ & $77(87.5)$ & \multirow{2}{*}{0.0053} & \multirow{2}{*}{$8.9(1.912-41.034)$} \\
\hline Enoxaparin & $126(58.9)$ & $2(1.6)$ & $124(98.4)$ & \\
\hline
\end{tabular}

Table 5: Statistical analysis per treatment group. Odds ratio for hemarthrosis requiring aspiration by anticoagulant.

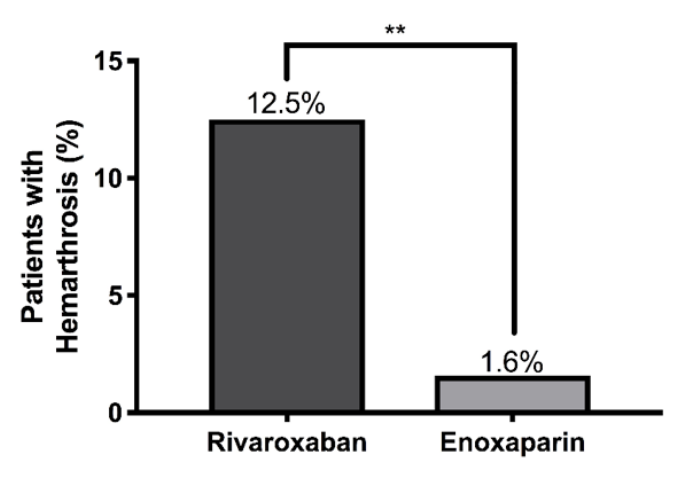

Figure 1: Percentage of patients requiring aspiration for hemarthrosis by anticoagulant.

treatment noted. Furthermore, the decision to prescribe prophylaxis by the physicians surveyed was largely based upon their personal clinical experience and understanding of the literature. The use of clinical practice guidelines was less often utilized when determining which patients to anticoagulate. As discussed previously, the AAOS has not provided guidelines for elective knee arthroscopic surgery chemoprophylaxis, and the American College of Chest Physicians does not currently recommend routine prophylaxis in low-risk patients.

\section{Anticoagulant mechanism of action}

LMWH medications are derived from heparin and their mechanism of action is the activation of anti-thrombin (Factor IIa). This interaction then inactivates Factor Xa in the coagulation cascade and exerts anticoagulant effects by preventing thrombosis and platelet activation. Due to its chemical properties, enoxaparin is administered through intravenous or subcutaneous routes [19]. In contrast, rivaroxaban is an oral, direct Factor Xa inhibitor with similar downstream anticoagulation effects as described above [20]. There are advantages to newer forms of chemoprophylaxis such as rivaroxaban in that they can be taken orally and many do not require laboratory monitoring.

\section{Brief literature review}

The incidence of DVT after arthroscopic knee surgery varies between studies and partially depends upon diagnostic technique (findings on clinical exam, ultrasound, venography, MR venography) with ranges between $0.02 \%$ to $41.2 \%$ [1-13]. Stringer., et al. were the first to report a $4.2 \%$ incidence of DVT after arthroscopic knee surgery in 1989, but routine prophylaxis after arthroscopy was not recommended [1]. Janssen., et al. reported PE incidence of $0.1 \%$. For patients at moderate risk for DVT/PE, most surgeons agree a prophylactic agent should be utilized [2-4]. This includes patients with two or more risk factors such as age greater than 40 years, obesity with a body mass index greater than $30 \mathrm{~kg} / \mathrm{m}^{2}$, current oral contraceptive use, hypercoagulopathy disorders or history of malignancy, personal or family history of DVT, nicotine use, and prolonged tourniquet time over 90 - 120 minutes. However, there is no consensus as to which prophylactic medication to administer and for what duration of prophylaxis.

Even though its use is controversial, thromboprophylaxis has been shown to reduce DVT rates in postoperative ACL reconstructed patients. Marlovits., et al. decreased their DVT incidence from $41.2 \%$ to $2.8 \%$ when prescribing $40 \mathrm{mg}$ enoxaparin given subcutaneously for 20 days for postoperative ACL reconstruction patients [21]. It should be noted however, that their protocol differs from common practice as their patients remained hospitalized for three to eight days postoperatively, and the mean surgical time was over 2 hours, possibly leading to their high incidence 
of magnetic resonance venography detection of DVT. Keller., et al. demonstrated that ten days of thromboprophylaxis was superior to five days after routine arthroscopy [22]. Wirth., et al. compared no thromboprophylaxis after knee arthroscopic procedures versus daily subcutaneous injections of 1750 IU reviparin (a LMWH) for seven to ten days. They found a decrease in their DVT incidence of $4.1 \%$ to $0.85 \%$ as detected by ultrasonography [23]. Camporese., et al. demonstrated a $72 \%$ relative risk reduction in DVT after knee arthroscopic surgery as diagnosed by ultrasonography in patients receiving nadroparin (a LMWH) for seven days postoperatively as compared to patients who only received graduated compression stockings. Michot., et al. performed a randomized controlled trial in patients after arthroscopic knee surgeries and randomized groups to prophylaxis with LMWH (weight-based dalteparin prescribed up to 30 days postoperatively) or placebo and decreased the DVT rate from $15.6 \%$ in patients in the placebo group to $1.5 \%$ in the LMWH group [24]. Many of these authors recommended routine use of LMWH thromboprophylaxis after orthopedic knee arthroscopic procedures [23,25]. However, there is currently no consensus on VTE prophylaxis after arthroscopic knee surgery.

\section{Current practices}

Today, rivaroxaban and enoxaparin are utilized for thromboprophylaxis with little literature available to guide practitioners in their use. Many studies cite the incidence of VTE in knee arthroscopy and ACL reconstruction [2] yet few have studied the specific complications related to various prophylactic medications.

\section{Bleeding risk}

In total knee and hip arthroplasty postoperative patients, rivaroxaban was found to have a non-significantly higher rate of bleeding as an adverse effect [26]. This data is difficult to extrapolate to elective sports medicine arthroscopic procedures in an ambulatory patient population.

Two studies have shown no significant difference in bleeding complications after prophylaxis with LMWH in postoperative knee arthroscopy patients when compared to no prophylaxis or the use of compression stockings only [21,25]. Wirth., et al. noted no major and 4 minor bleeding complications in postoperative knee arthroscopy patients when prescribing reviparin (a LMWH), however, this was not statistically significant. Michot., et al. noted twice the amount of minor bleeding complications in their patients using LMWH (12\% versus 6\%) plus two patients in the LMWH group re- quired aspiration with a resultant diagnosis of hemarthrosis [24]. The ERIKA randomized controlled trial showed that rivaroxaban $10 \mathrm{mg}$ once daily for seven days was effective at preventing VTE in postoperative knee arthroscopy patients and did not exhibit significant adverse bleeding [27]. A recent study randomized patients to either LMWH (bemiparin 3500 IU injected daily) or rivaroxaban $10 \mathrm{mg}$ taken orally daily. Each medication was prescribed for three weeks to 467 moderate-severe risk patients who underwent arthroscopic knee surgery. The authors noted no DVT or PE and only one episode of a minor bleeding complication in a patient with epistaxis that resolved after discontinuing rivaroxaban [28].

To our knowledge, no study has directly evaluated the incidence of hemarthrosis in postoperative sports medicine patients when comparing rivaroxaban and enoxaparin prophylaxis. Knee effusion and/or hemarthrosis can cause pain, delay in physical therapy, knee stiffness, and the potential need for further surgery. Hence, preventing knee effusion post-arthroscopy enables more appropriate progress in the rehabilitation phase, is cost effective, and results in overall less pain for the patient.

The current study demonstrates that the odds of requiring aspiration after thromboprophylaxis with rivaroxaban is 8.9 times greater than with enoxaparin. This study highlights a potentially higher intra-articular bleeding risk with prophylactic anticoagulant therapy in the ambulatory sports medicine surgical patient. Additionally, there were no symptomatic DVTs identified, indicating that both enoxaparin and rivaroxaban are likely effective agents for postoperative thromboprophylaxis in the postoperative sports medicine patient.

Compliance and cost effectiveness are additional issues surrounding medications for VTE prophylaxis. Patient compliance is difficult to monitor and measure; however, it is presumed that patients may better tolerate an oral medication as opposed to daily subcutaneous injections.

\section{Limitations of the Study}

There are multiple limitations of the current study. Firstly, the study performed was observational and retrospective in nature across two defined periods of postoperative anticoagulation protocols. Thereby, this study also lacks comparison to a control group (no anticoagulation), aspirin, and/or mechanical prophylaxis. Secondly, this study was not blinded; the anticoagulant chosen dur- 
ing the treatment period was not blinded to the examiner during routine postoperative care. This study is further limited by a small sample size, however, the results were statistical significant and our a priori power analysis sample sizes were met. Finally, there exists a selection bias in the patient cohorts as patients who are likely to undergo arthroscopic knee surgery tend to be younger, more active, and therefore are generally healthier. Our cohorts were fairly homogeneous when comparing across intervention groups and no statistical significance was found when comparing their demographic data, but it should be noted that the majority of patients were healthy (American Society of Anesthesiologists [ASA] class 1) males under the age of 40 years (Table 2). As such, this selection bias may limit the generalizability of the results presented here to other patient populations. Future prospective studies as well as a well-defined risk stratification recommendation would better improve the orthopedic sports medicine physician's decision-making process regarding routine postoperative thromboprophylaxis.

The current study did not aspirate asymptomatic postoperative effusions to evaluate for hemarthrosis as these effusions were felt to not be clinically significant during postoperative rehabilitation; this may have falsely decreased the total incidence of hemarthrosis. Similarly, this study did not evaluate for asymptomatic DVT with imaging studies.

Finally, patient compliance with anticoagulation protocols was not documented in this study. However, clinical wisdom suggests that patients may tolerate oral medication better than daily subcutaneous injections. This could theoretically lead to a decrease in the number of patients adequately anticoagulated in the enoxaparin group. If patients were more likely to discontinue a medication requiring an injection as opposed to an oral medication, this could lead to a decrease in the incidence of hemarthrosis seen in the enoxaparin group.

\section{Conclusion}

In conclusion, if a surgeon chooses to prescribe chemoprophylaxis for ambulatory postoperative sports medicine patients, this study supports consideration of avoiding rivaroxaban, given the higher risk of a symptomatic hemarthrosis in the early postoperative period. A better choice may be enoxaparin as it is associated with lower postoperative hemarthrosis complication risk and appears to be safe in the immediate postoperative period for patients undergoing common orthopedic sports medicine procedures. Fur- ther studies are required to determine the optimal prophylactic anticoagulant, dose, and duration of thromboprophylaxis for the outpatient orthopedic sports medicine postoperative patient with and without VTE risk factors. Future studies may focus on the development of a knee arthroscopy-specific VTE risk scoring system to assist sports medicine surgeons in VTE prevention.

\section{Funding Support}

There was no internal nor external funding provided for this study.

\section{Conflicts of Interest}

The authors declare no conflicts of interest related to this study.

\section{Ethics Approval}

All procedures performed in studies involving human participants were in accordance with the ethical standards of the institutional and/or national research committee (Baylor College of Medicine Institutional Review Board, Protocol H-44966) and with the 1964 Helsinki declaration and its later amendments or comparable ethical standards.

\section{Author Contributions}

All authors contributed significantly to the concept, analysis, writing, revision, and approval of this manuscript.

\section{Bibliography}

1. Stringer MD., et al. "Deep vein thrombosis after elective knee surgery. An incidence study in 312 patients". The Journal of Bone and Joint Surgery British 71 (1989): 492-497.

2. Janssen RP., et al. "Arterial complications, venous thromboembolism and deep venous thrombosis prophylaxis after anterior cruciate ligament reconstruction: A systematic review". World Journal of Orthopaedics 7 (2016): 604-617.

3. Krych AJ., et al. "Incidence and Risk Factor Analysis of Symptomatic Venous Thromboembolism After Knee Arthroscopy". Arthroscopy 31 (2015): 2112-2118.

4. Falck-Ytter Y., et al. "Prevention of VTE in orthopedic surgery patients: Antithrombotic Therapy and Prevention of Thrombosis, 9th edition: American College of Chest Physicians Ev- 
idence-Based Clinical Practice Guidelines". Chest 141 (2012): e278S-e325S.

5. Ye S., et al. "The incidence of deep venous thrombosis after arthroscopically assisted anterior cruciate ligament reconstruction". Arthroscopy 29 (2013): 742-747.

6. Erickson BJ., et al. "Rates of Deep Venous Thrombosis and Pulmonary Embolus After Anterior Cruciate Ligament Reconstruction: A Systematic Review". Sports Health 7 (2015): 261266.

7. Demers C., et al. "Incidence of venographically proved deep vein thrombosis after knee arthroscopy". Archives of Internal Medicine 158 (1998): 47-50.

8. Jaureguito JW., et al. "The incidence of deep venous thrombosis after arthroscopic knee surgery". American Journal of Sports Medicine 27 (1999): 707-710.

9. Maletis GB., et al. "Incidence of symptomatic venous thromboembolism after elective knee arthroscopy". The Journal of Bone and Joint Surgery American 94 (2012): 714-720.

10. Cullison TR., et al. "The incidence of deep venous thrombosis in anterior cruciate ligament reconstruction". Arthroscopy 12 (1996): 657-659.

11. Schippinger G., et al. "Thromboembolic complications after arthroscopic knee surgery. Incidence and risk factors in 101 patients". Acta Orthopaedica Scandinavica 69 (1998): 144-146.

12. Gaskill T., et al. "The Prevalence of Symptomatic Deep Venous Thrombosis and Pulmonary Embolism After Anterior Cruciate Ligament Reconstruction". American Journal of Sports Medicine 43 (2015): 2714-2719.

13. Graham WC and Flanigan DC. "Venous thromboembolism following arthroscopic knee surgery: a current concepts review of incidence, prophylaxis, and preoperative risk assessment". Sports Medicine 44 (2014): 331-343.

14. Delis KT., et al. "Incidence, natural history and risk factors of deep vein thrombosis in elective knee arthroscopy". Thrombosis and Haemostasis 86 (2001): 817-821.

15. Bogunovic L M., et al. "The role of deep venous thrombosis prophylaxis after anterior cruciate ligament reconstruction".
Operative Techniques in Sports Medicine 24 (2015): 73-76.

16. Jacobs JJ., et al. "American Academy of Orthopaedic Surgeons clinical practice guideline on: preventing venous thromboembolic disease in patients undergoing elective hip and knee arthroplasty". The Journal of Bone and Joint Surgery American 94 (2012): 746-747.

17. Sturgill LP., et al. "Interrater reliability of a clinical scale to assess knee joint effusion". Journal of Orthopaedic and Sports Physical Therapy 39 (2009): 845-849.

18. Keller RA., et al. "Deep Venous Thrombosis Prophylaxis in Anterior Cruciate Ligament Reconstructive Surgery: What Is the Current State of Practice?" Sports Health 10 (2018): 156-159.

19. Hirsh J., et al. "Heparin and low-molecular-weight heparin: mechanisms of action, pharmacokinetics, dosing, monitoring, efficacy, and safety". Chest 119 (2001): 64S-94S.

20. Samama MM. "The mechanism of action of rivaroxaban--an oral, direct Factor Xa inhibitor--compared with other anticoagulants". Thrombosis Research 127 (2011): 497-504.

21. Marlovits S., et al. "Extended-duration thromboprophylaxis with enoxaparin after arthroscopic surgery of the anterior cruciate ligament: a prospective, randomized, placebo-controlled study". Arthroscopy 23 (2007): 696-702.

22. HKSIKF. "Thromboseprophylaxe in der ambulanten kniechirurgie - Untersuchung an uber 2000 arthroskopierten patienten". Orthop Praxis 10/95 (1995): 681-683.

23. Wirth T., et al. "Prevention of venous thromboembolism after knee arthroscopy with low-molecular weight heparin (reviparin): Results of a randomized controlled trial". Arthroscopy 17 (2001): 393-399.

24. Michot M., et al. "Prevention of deep-vein thrombosis in ambulatory arthroscopic knee surgery: A randomized trial of prophylaxis with low--molecular weight heparin". Arthroscopy 18 (2002): 257-263.

25. Camporese G., et al. "Low-molecular-weight heparin versus compression stockings for thromboprophylaxis after knee arthroscopy: a randomized trial”. Annals of Internal Medicine 149 (2008): 73-82. 
26. Lassen MR., et al. "The effects of rivaroxaban on the complications of surgery after total hip or knee replacement: results from the RECORD programme". The Journal of Bone and Joint Surgery. British 94 (2012): 1573-1578.

27. Camporese G., et al. "Efficacy of Rivaroxaban for thromboprophylaxis after Knee Arthroscopy (ERIKA). A phase II, multicentre, double-blind, placebo-controlled randomised study". Thrombosis and Haemostasis 116 (2016): 349-355.

28. Munoa L., et al. "Rivaroxaban is as efficient and safe as bemiparin as thromboprophylaxis in knee arthroscopy". Musculoskeletal Surgery 98 (2014): 21-25.

Volume 4 Issue 11 November 2021

(C) All rights are reserved by Brian P Davis., et al. 